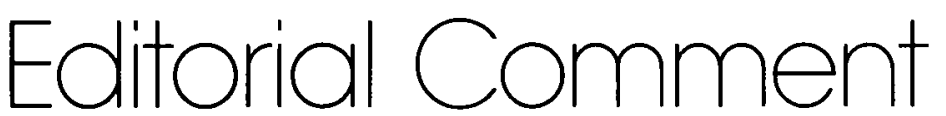

Martin S. Favero, PhD

\title{
Recommended Precautions for Patients Undergoing Hemodialysis Who Have AIDS or Non-A, Non-B Hepatitis
}

\begin{abstract}
Precautions used for dialysis patients who have acquired immunodeficiency syndrome or non-A, non-B hepatitis are based on infection control strategies developed for the control of hepatitis $B$ in dialysis centers. Specificially, these recommendations include identifying infected patients; isolating (except for non$A$, non-B hepatitis patients) patients, and separating staff, and equipment; applying blood precautions and aseptic techniques; and using good environmental control procedures. AIDS patients can be safely dialyzed by either hemodialysis or peritoneal dialysis in hospitalbased or free-standing centers, or at home without risk of AIDS transmission to other patients or to staff members, if precautions that have been developed for the control of hepatitis $B$ infection in dialysis units are employed. Further, the type of dialysis treatment, or modality, should be based on the needs of the patient and not on a fear of risk of disease transmission. [Infect Control 1985; 6(8):301-305.]
\end{abstract}

\section{INTRODUCTION}

While the major emphasis of this article is on recommended precautions for AIDS patients undergoing hemodialysis, precautions for non-A, non-B patients undergoing hemodialysis are also discussed because of confusion in the nephrology community about strategies for controlling this infection in dialysis centers.

The number of dialysis centers, patients, and staff has increased significantly in the US in the past 15 years. The Centers for Disease Control (CDC) began conducting

From the Hospital Infections Program, Center for Infectious Diseases, Centers for Disease Control, Allanta, Georgia.

Portions of this paper were presented at the workshop, "Infectious Diseases and Artificial Organs With an Emphasis on AIDS and Hepatitis," held at the American Society for Artificial Internal Organs (ASAIO) May 4, 1984, Washington, D.C., and at the International Conference on Acquired Immunodeficiency Syndrome, April 16, 1985, Allanta, Georgia.

Address reprinl requests to Martin S. Favero, PhD, Chief, Nosocomial Infections Laboratory Branch, Hospital Infections Program, Center for Infectious Diseases, Centers for Disease Control, Allanta, GA 30333. surveillance on viral hepatitis in dialysis centers in 1969 and found that between 1962 and 1974 the incidence of hepatitis B surface antigen (HBsAg) positivity among patients and staff more than doubled to $6.2 \%$ and $5.2 \%$, respectively. ${ }^{1}$ In a separate survey of 15 hemodialysis centers in 1973, Szmuness et al found that the prevalence of HBsAg positivity was $16.8 \%$ among patients and $2.4 \%$ among staff. ${ }^{2}$ In 1976, CDC's surveillance system was expanded by collaboration with the Health Care Financing Administration (HCFA). During HCFA's annual facility survey a questionnaire on viral hepatitis, infection control practices, and disinfection and sterilization strategies was included for CDC. The results of these surveillance studies showed that the incidence and prevalence of $\mathrm{HBsAg}$ positivity among patients and staff had decreased significantly since 1976 and that this reduction appeared to be due to better infection control practices, which included complete segregation of $\mathrm{HBsAg}$-positive patients and their dialysis equipment in conjunction with rapid identification by serologic surveillance of patients who seroconvert to $\mathrm{HBsAg}$ positivity. ${ }^{3}$ From 1976 to 1982 , the incidence of $\mathrm{HBsAg}$ positivity among patients decreased from $3 \%$ to $0.5 \%$ and among staff members from $2.6 \%$ to $0.4 \%$. In 1985 , the average hemodialysis center in the US, although still considered a high-risk environment for acquiring hepatitis $\mathrm{B}$ virus (HBV) infection, is no longer considered to be an "extraordinary" high-risk environment. ${ }^{4}$

The purpose of this article is to discuss those infection control strategies and precautions that have been shown to reduce the incidence of hepatitis $B$ infection among dialysis patients and staff members as a means of formulating specific infection control practices and precautions that would be effective when used for dialyzing patients who have a diagnosis of acquired immunodeficiency syndrome (AIDS) or non-A non-B hepatitis.

\section{AIDS}

The similarities in the epidemiologic characteristics of AIDS and hepatitis B suggest that measures known to be effective in preventing the spread of hepatitis B may also be effective in preventing transmission of AIDS. ${ }^{5-7}$ As 
mentioned above, the incidence and prevalence of hepatitis B infection among patients and staff members in dialysis centers in the US decreased significantly between 1976 and 1982, and these reductions are based on infection control strategies that include: ${ }^{8,4}$

1. Identifying $\mathrm{HBsAg-positive} \mathrm{patients.}$

2. Isolating $\mathrm{HBsAg}$-positive patients, including the use of designated staff and equipment in isolation areas.

3. Restricting non-disposable supplies to individual patients unless sterilized between uses.

4. Applying blood precautions and aseptic techniques.

5. Cleaning and disinfecting dialysis equipment, especially surfaces that are frequently touched during operations and/or contaminated with blood.

Currently, there is no evidence that AIDS has been transmitted in the dialysis center environment, either from patient to patient or patient to staff member. However, since AIDS does appear to be transmitted in the same manner as hepatitis B (ie, bloodborne) it would be prudent to assume that AIDS has the potential for being transmitted in dialysis centers and to use the same strategies for infection control as well as sterilization and disinfection that are used for hepatitis B in dialysis units. It is emphasized that this approach is a most conservative one and justified primarily on the severity and grave consequences of AIDS infection. There is little doubt that hepatitis B infection is transmitted with much more efficiency than is AIDS in the same type of health care environment. This probably is due to the accumulating evidence that the titer of the presumed agent of AIDS, the retrovirus, HTLV-III/LAV, is not as high as $\mathrm{HBV}$ in blood of infected patients and/or that high numbers of the HTLV-III/LAV virus are needed to produce infection. Currently, CDC is conducting a needlestick study in which health professionals who have experienced percutaneous or mucus membrane exposure from a documented AIDS patient are followed for 3 years. While this study has not been completed, preliminary results have shown that among 326 health care workers followed, none has developed AIDS, and a subset of 40 health care workers who have been tested for seroconversion to antibody to HTLV-III/LAV (anti-HTLV-III) have all been negative. ${ }^{9.10}$ By comparison, Seeff and co-workers showed that health professionals who were recipients of needlesticks from individuals known, to be $\mathrm{HBsAg}$ positive and $\mathrm{HBeAg}$ positive (a serologic marker indicating active $\mathrm{HBV}$ replication and high $\mathrm{HBV}$ titer in serum) experienced infection rates of $37 \% .^{11}$

It is known by infectivity studies that the HBV titer in serum of a person who is $\mathrm{HBsAg}$ and $\mathrm{HBeAg}$ positive is approximately $10^{8}$ infectious doses per $\mathrm{ml}$. Although similar infectivity studies with HTLV-III/LAV have not been completed there were reports and discussions at the International Conference on AIDS that patients diagnosed with AIDS and who are anti-HTLV-III positive had titers of approximately $10^{2}$ to $10^{4}$ infectious doses per $\mathrm{ml}$ of sera. The natural; history of this virus in humans and its concentrations in blood, serum, and various secretions and excretions in infected patients are not yet known but should be clarified in the near future when the results of major studies of several groups in the US are completed.
In any event it does appear that the efficiency of AIDS transmission from patient to patient or patient to staff member is significantly less than hepatitis $B$ so that if the types of infection control practices that have significantly reduced the incidence and prevalence of hepatitis $B$ among patients and staff members in dialysis units are practiced, the risk of AIDS transmission can be reduced significantly.

Unfortunately, there is a tendency on the part of dialysis staff members and other health care professionals to refuse to admit AIDS patients to hemodialysis centers, insist that they be dialyzed at home, and/or recommend that the mode of dialysis be peritoneal dialysis rather than hemodialysis.

These ultraconservative approaches are based on the grave consequences of AIDS infection and the assumptions that AIDS can be easily transmitted. There are no epidemiologic or laboratory data that support these assumptions of efficient disease transmission. The modality of dialysis should not be influenced by unfounded fears of AIDS transmission to other patients or staff within the dialysis center environment; all indications are that AIDS patients can be safely dialyzed.

At the time of the ASAIO Workshop in May 1984, participants estimated that there were no more than 10 patients with a diagnosis of AIDS who were being dialyzed in chronic hemodialysis centers in the US. Approximately 1 year later in May 1985 TKS Rao and coworkers reported that they had dialyzed 26 AIDS patients in Brooklyn, NY. ${ }^{12}$ In the last 12 months CDC has received an increasing number of letters and telephone inquiries on infection control strategies for handling AIDS patients who require dialysis. In June 1985 the reported number of AIDS cases to CDC since 1981 reached approximately 10,000 and it is estimated that an additional 10,000 may be reported in the next 12 months. It is clear that the number of patients presenting for chronic hemodialysis will increase. These patients will include those who have clinical cases of AIDS, or AIDSrelated complex, as well as patients who are anti-HTLVIII positive without clinical signs or symptoms of AIDS but who must be considered infectious.

The following infection control strategies, which are derived from those that have been shown to be effective in the control of hepatitis B infections in dialysis centers, can be applied to AIDS patients who are treated by hemodialysis or peritoneal dialysis in free-standing or hospitalbased dialysis centers. Often, AIDS patients who require dialysis are seriously ill. Dialysis is performed on an acute basis in hospital intensive care units or in hospital-based dialysis centers. However, there probably will be an increasing number of patients who have a diagnosis of AIDS but who are ambulatory and have end-stage renal disease as well as individuals who have end-stage renal disease and who are anti-HTLV-III positive.

Specific Infection Control Strategies and Precautions for AIDS Patients Undergoing Dialysis

1. Identification of patients who are suspected of having AIDS.

For purposes of infection control in the dialysis center an individual with AIDS is defined as one who has been diagnosed based on clinical signs or symptoms 
that indicate AIDS infection or are compatible with the AIDS-related complex of infection, and/or is positive for anti-HTLV-III. As mentioned previously, the form of dialysis undertaken, ie, hemodialysis or peritoneal dialysis, should be determined by the medical needs of the patient and while a diagnosis of AIDS may influence that decision, the risk of disease transmission to patients and staff should not be a significant factor determining the type of dialysis.

2. Isolation of AIDS patients into separate rooms, segregation of staff, and dialysis equipment.

In a dialysis center, admission of an AIDS patient could result in having four cohorts of patients that need to be considered for infection control strategies:

a. HBsAg-negative-AIDS-negative (most of the patient population),

b. HBsAg-positive-AIDS-negative (the next most frequent patient population),

c. HBsAg-negative-AIDS-positive, and

d. HBsAg-positive-AIDS-positive.

In most dialysis centers in the US it is virtually impossible to maintain four separate dialysis treatment areas for each of the groups described above. In fact, in most centers that have a separate area where $\mathrm{HBsAg}$ positive patients are dialyzed on separate machines, it would be difficult if not impossible to designate an additional separate area for the dialysis of one or more AIDS patients. Further, in some centers, the designation of a particular dialysis machine for exclusive use by an AIDS patient is a significant problem for the center. In the context of these logistical constraints the following range of infection control strategies are offered to centers when AIDS patients present for dialysis:

a. Separate area-separate machines.

This strategy has been shown to be very effective in reducing the incidence of hepatitis $B$ infection in dialysis units and if space is available it is an easy and effective means of preventing AIDS transmission. However, for reasons mentioned previously, the efficiency of AIDS transmission in the dialysis environment is much less than hepatitis B so that this particular infection control strategy, using a separate room and separate designated machines, becomes an ultra-conservative infection control practice and one that would be feasible only if adequate space and staff are available.

b. Designated area-separate machine.

This strategy implies the use of a separate machine at a particular site in a dialysis center where $\mathrm{HBsAg}$ negative patients are dialyzed. This approach is also conservative but one that would be effective if it can be logistically practiced in the center.

c. Separate machine.

The use of a separate machine along with proper blood precautions and environmental control would be a conservative and effective approach to prevent AIDS transmission from patient to patient and patient to staff members. The use of a separate machine for dialyzing an $\mathrm{HBsAg}$ positive patient or an AIDS patient in this type of an infection control program does not mean that the dialysis machine cannot be used when it becomes available for dialysis of other patients. The risk of disease transmission, for hepatitis B as well as AIDS, is not associated with the internal circuit of the artificial kidney machine but rather with the frequently touched environmental surfaces of the machine and surfaces of ancillary equipment in the immediate area. Thus, the proper use, daily cleaning, and disinfection of these surfaces are important elements in the infection control program.

The conventional cleaning and disinfection procedures used for the internal circuits of an artificial kidney machine are designed to control gram-negative bacterial contamination and are adequate for both the control of hepatitis B as well as AIDS. ${ }^{4}$ There are no added environmental control steps, or disinfection procedures or decontamination protocols for dialysis machines that have been used to dialyze patients with AIDS or hepatitis. Consequently, the availability of dialysis machines and space will primarily dictate specific infection control strategies listed above, but it is emphasized that the minimum strategy, ie, the use of a separate machine, would be considered an effective and conservative strategy for preventing AIDS transmission.

Some dialysis centers have used alternate approaches. For example, some AIDS patients have been dialyzed in the privacy of a hospital room; this may be necessary when a patient is gravely ill or in intensive care but may not be a feasible approach if the patient is ambulatory.

When the medical needs of the patient indicate, peritoneal dialysis can be employed. This can be in the form of either continuous ambulatory peritoneal dialysis (CAPD) or intermittent cyclic peritoneal dialysis (ICPD). These dialysis modalities are more often performed at home than in dialysis centers or hospitals. Bags containing peritoneal dialysis fluid should be be handled with care but extraordinary precautions are not necessary. In the home environment one should handle bags containing peritoneal dialysis fluid with a disposable glove and the contents can be carefully poured down a toilet. The empty bag should be wrapped securely in a plastic bag, and bagged once again and discarded in the conventional trash system. In the dialysis center peritoneal dialysis bags can be disposed of in the same fashion as other solid waste associated with peritoneal dialysis.

3. Restriction of nondisposable supplies to individual AIDS patients unless sterilized between uses.

4. Hemodialyzers should not be reused with AIDS patients.

5. Application of blood precautions and aseptic techniques.

Proper use of gloves, gowns and handwashing techniques for the control of hepatitis $B$ in dialysis centers have been described and will not be detailed here. ${ }^{4.8}$ It is emphasized, however, that extraordinary use of protective clothing, face shields, goggles, etc. is not warranted, and that precautions used for the control of hepatitis $B$, which are conservative in themselves, become most conservative when applied to the control 
of AIDS in a dialysis center.

6. Cleaning and disinfection of dialysis machines, other equipment, and environmental surfaces that are frequently touched by staff members and patients.

Procedures for environmental control and for disinfection and sterilization of hemodialysis systems have been described. ${ }^{4}$ Procedures for the disinfection of the fluid pathway of individual dialysis machines can include sodium hypochlorite, formaldehyde, or glutaraldehyde formulations, all of which are intended primarily for the control of bacterial contamination; any of these germicides would be appropriate for machines that are used for dialyzing AIDS patients. It is the external surfaces of dialysis systems, especially those that are frequently touched, such as control knobs, that are of concern in preventing cross-contamination. As with procedures designed for the control of hepatitis B these surfaces can be cleaned using a clean cloth soaked with an appropriate chemical germicide such as sodium hypochlorite solutions containing approximately 500 to $1000 \mathrm{ppm}(\mathrm{mg} / \mathrm{L})$ free chlorine or any other appropriate intermediate to high level disinfectant to spot clean. ${ }^{13}$ Extraordinary use of disinfectants is not indicated. Blood spills, contaminated surfaces, etc; can basically be managed in the same way that has been described for $\mathrm{HBV}$ contamination. This means that conventional sterilization and disinfectant regimens can be used for medical devices; for example, steam sterilizers, ethylene oxide gas sterilizers, and high level disinfectants can be used in the same manner that they are ordinarily used in hemodialysis centers or other health care environments. ${ }^{4,13}$

It has become increasingly evident that the AIDS agent, HTLV/III-LAV is relatively sensitive to chemical germicides and heat. ${ }^{14-17}$ In fact it appears that conventional intermediate and high level disinfectants that are approved for use in hospitals by the US Environmental Protection Agency would be effective against HTLV/III-LAV.

\section{NON-A, NON-B HEPATITIS}

In the US, there are at least two types of non-A, non-B (NANB) hepatitis that are bloodborne and potentially could be transmitted in a hemodialysis environment. ${ }^{18-20}$ The diagnosis of NANB hepatitis remains one of exclusion and depends on testing for serologic markers of acute hepatitis A and hepatitis B infection and, ideally, cytomegalovirus and Epstein-Barr virus as well. If clinical signs and symptoms are consistent with viral hepatitis and not associated with other causes of liver injury or infections, then a presumptive diagnosis of NANB hepatitis can be made. In 1982, Alter et al determined that the incidence of NANB hepatitis among patients in dialysis centers was $1.6 \%$ and in $1983,1.8 \%{ }^{3}$ At least one outbreak of NANB hepatitis in a hemodialysis center has been reported in the literature. ${ }^{21}$

Strategies for infection control for hemodialysis patients with NANB hepatitis differ from those applied to patients with hepatitis B virus (HBV) infection. Since there is no specific diagnostic test for NANB hepatitis, it is difficult to implement a rational patient isolation and segregation protocol similar to that devised for hepatitis $\mathbf{B}$; in all probability any such efforts would group together patients with different etiologic agents and mix patients who are susceptible to NANB hepatitis with patients who are infected with NANB hepatitis virus. Furthermore, the transmission of NANB hepatitis in a dialysis environment may not be as efficient as that of $\mathrm{HBV}$, possibly because patients with NANB hepatitis have a lower titer of virus in the blood (generally, $10^{3} / \mathrm{ml}$ or less) than do patients who are $\mathrm{HBsAg}$-positive (up to $10^{8} / \mathrm{ml}$ ). ${ }^{20,22}$ The existence of an asymptomatic carrier state for NANB hepatitis has been confirmed by both animal and human studies. ${ }^{23-25}$ However, the inability to detect that state means there are no available methods to determine when isolation of a patient who has had or is suspected of having NANB hepatitis is no longer needed.

Because of these limitations, it is not recommended that hemodialysis patients suspected of having NANB hepatitis be segregrated in a separate room or that they receive dialysis on separate machines. Rather, it is recommended that appropriate blood precautions be taken and scrupulous aseptic technique practiced. Particular attention should be paid to the cleaning and disinfection of instruments, machines and environmental surfaces that are routinely touched. As is recommended for all hemodialysis patients, no articles should be shared between patients. ${ }^{26,8}$ If such sharing is necessary, items should be autoclaved between use.

\section{REFERENCES}

1. Snydman D, Bryan J, Hanson B: Hemodialysis-associated hepatitis in the United States-1972. J Infect Dis 1975; 132:109-113.

2. Szmuness W, Prince AM, Grady GF, et al: Hepatitis B infection. A point prevalence study in 15 U.S. hemodialysis centers. JAMA 1974; 227:901-906.

3. Alter MJ, Favero MS, Petersen NJ, et al: National surveillance of dialysisassociated hepatitis and other diseases. Dialysis Transplant 1983b; 12:860-868

4. Favero MS: Dialysis-associated diseases and their control, in, Bennett JV, Brachman PS (eds): Hospital Infections, ed 2. Boston, Massachusetts, Litule, Brown and Company Inc., 1985.

5. Prevention of acquired immune deficiency syndrome (AIDS): Report of interagency recommendations. MMWR 1983; 32:101-103.

6. Acquired immune deficiency syndrome (AIDS): Precautions for clinical and laboratory staffs. $M M W R$ 1982; $31: 577-580$.

7. Quinn TC: Precautions for patients hospitalized with acquired immunodeficiency syndrome. Infect Control 1983; 4:79-80.

8. Hepatitis-Control measures for hepatitis B in dialysis centers. Viral Hepatitis: Investigation and Control Series - November 1977, HEW Publication No. (CDC) 78-8358

9. Update: Prospective evaluation of health-care workers exposed via the parenteral or mucous-membrane route to blood or body fluids from patients with acquired immunodeficiency syndrome-United States. MMWR 1985 ; 34:101-103.

10. McCray E, Winslow N, Solomon S, et al: Prospective evaluation of health-care workers with parenteral or mucous-membrane exposure to blood from patients with acquired immunodeficiency syndrome, United States: An Update. Presented at the International Conference on Acquired Immunodeficiency Syndrome (AIDS) April 16, 1985, Atlanta, Georgia. Centers for Disease Control, Atlanta. Abstract - $p 45$.

11. Seeff LB, Wright EC, Zimmerman HI , et al: Type B hepatitis after needlestick exposure: Prevention with hepatitis immune globulin. Ann Intern Med 1978; 88:285-293.

12. Rao TKS, Manis T, Friedman EA: Dismal prognosis despite maintenance hemodialysis in AIDS nephropathy and chronic uremia. Abstracts of the American Society for Artificial Internal Organs, Inc. May 9, 1985, Atlanta Georgia. American Society for Artificial Internal Organs, Boca Raton, Florida. Abstract p 47.

13. Favero MS: Sterilization, disinfection, and antisepsis in the hospital, in, Manual of Clinical Microbiology, ed 4, American Society for Microbiology, Washington, DC, 1985; $952-959$.

14. McDougal SJ. Cort SP, Kennedy MS, et al: Immunoassay for the detection of infectious human retrovirus, lymphadenopathy-associated virus (LAV) $J$ 
Immunol Methods 1985: 76:171-183

15. Martin LS, McDougal JS, Loskoski SL: Disinfection and inactivation of human T Lymphotropic Virus Type III/Lymphadenopathy Virus./Infect Dis 1985; to be published.

16. Spire B, Barre-Sinoussi F, Montagnier L, et al: Inactivation of Lymphadenopathy associated virus by chemical disinfectants. Lancet October 20, 1984; 899-901.

17. Spire B, Dormont D, Barre-Sinoussi F, et al: Inactivation of Lymphadenopathy-Associated Virus by heat, gamma rays, and ultraviolet light. Lancet january 26,$1985 ; 188-189$.

18. Tabor E, April M, Seef LB, et al: Acquired immunity to human non-A, non-B hepatitis: Cross-challenge of chimpanzees with three infectious human sera. $J$ Infect Dis 1979; 140(5):789-793.

19. Hollinger FB, Mosley JW, Szmuness W, et al: Transfusion-transmitted viruses study: Experimental evidence for two non-A, non-B hepatitis agents. $J$ Infect Dis 1980; 142(3):400-407.

20. Bradley DW, Maynard JE, Popper $H$, et al: Postransfusion non-A, non-B hepatitis: Physicochemical properties of two distinct agents. J Infect Dis 1983 148(2):254-265.

21. Galbraith RM, Dienstag JL, Purcell RH, et al: Non-A, non-B hepatitis associated with chronic liver disease in a hemodialysis unit. Lancet 1979; $951-953$

22. Bradley DW: Transmission, etiology, and pathogenesis of viral hepatitis nonA, non-B in non-human primates, in, Chisari FV (ed): Advances in Hepatitis Research. New York, Masson Publishing USA lnc. 1984, pp 268-280.

23. Alter $\mathrm{HJ}$, Purcell RH, Holland $\mathrm{PV}$, et al: 'Transmissible agent in non-A, non- $\mathrm{B}$ hepatitis. Lancet 1978; 459-463.

24. Tabor E, Gerety RJ, Drucker JA, et al: Transmission of non-A, non-B hepatitis from man to chimpanzee. Lancet 1978; 463-465.

25. Tabor E, Seef Lb, Gerety RJ : Chronic non-A, non-B hepatitis carrier statetransmissible agent documented in one patient over a six-year period. $N E$ ElJ Med 1980; 303:140-143.

26. Alter $M J$, Ahtone J, Maynard JE: Hepatitis B virus transmission associated with a multiple-dose vial in a hemodialysis unit. Ann Intern Med 1983; 99(3):330-333. 